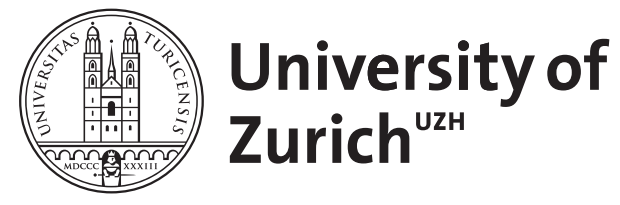

Zurich Open Repository and Archive

University of Zurich

University Library

Strickhofstrasse 39

CH-8057 Zurich

www.zora.uzh.ch

Year: 2010

Transformation of evidence to knowledge: a neglected task within the medical profession

Steurer, J

DOI: https://doi.org/10.1007/s00256-009-0812-9

Posted at the Zurich Open Repository and Archive, University of Zurich ZORA URL: https://doi.org/10.5167/uzh-31508

Journal Article

Published Version

Originally published at:

Steurer, J (2010). Transformation of evidence to knowledge: a neglected task within the medical profession. Skeletal Radiology, 39(1):1-3.

DOI: https://doi.org/10.1007/s00256-009-0812-9 


\title{
Transformation of evidence to knowledge: a neglected task within the medical profession
}

\author{
Johann Steurer
}

Published online: 11 October 2009

(C) ISS 2009

The term evidence-based medicine (EBM) was first used in a paper published in JAMA in 1992 [1]. In the book Evidence-based medicine: how to practice and teach EBM, EBM was defined as the "conscientious, explicit, and judicious use of current best evidence in making decisions about the care of individual patients" [2]. This was not a new concept at the time. Physicians caring for patients before the era of EBM presumably also made use of the best available evidence. What made EBM a new paradigm for medical practice was the description of four steps that physicians should follow in order to practice evidencebased medicine: (1) physicians should formulate a specific and clinically relevant question and then (2) quickly and effectively search for the best available evidence. (3) Once they have critically appraised the validity and utility of the evidence, (4) they should apply the findings of their critical appraisal in clinical practice.

The idea of EBM disseminated rapidly throughout the world; new institutions were established, and specialized EBM journals were launched. There are two reasons for this rapid dissemination: first, the tremendous increase in the number of published papers, and second, the decline in trust toward 'experts' and authority. Up until half a century ago, only a few clinical trials measuring clinically relevant outcomes had been produced. Since then, more than 100,000 randomized controlled trials have been published; approximately 12,000 were published in 2008 alone. Furthermore, the results of several cohort, case-control, and other types of studies lower on the EBM hierarchy have

\footnotetext{
J. Steurer $(\square)$

Horten Centre for Patient Oriented Research and Knowledge

Transfer,

Raemistrasse 100

8091 Zürich, Switzerland

e-mail: johann.steurer@usz.ch
}

been reported. It has become quite impossible for a physician to identify all of the relevant papers for his or her specialty, not to mention to read them, in order to keep up to date. The intention of the founders of EBM was to enable physicians to identify relevant research articles and to critically appraise the original literature with the hope of making them more independent of the opinions and beliefs of the prevailing experts.

The rank and high standing of medical experts was anathema to the founders of EBM. Medical experts are those who state their beliefs and opinions at medical conferences and in review articles. Among the weaknesses that they may fall prey to are the selective citing of research results, inappropriate judgments regarding the methodological quality of cited papers, and the formulation of conclusions and recommendations that are not supported by the available evidence [3]. Furthermore, suspicions that financial relationships between experts and pharmaceutical companies might have an impact on the conclusions and recommendations reached by experts is common and not entirely unwarranted. Stelfox et al. showed that experts who recommend calcium-channel antagonists for the treatment of high blood pressure are significantly more likely than neutral authors to have financial ties with manufacturers of these drugs [4]. Not surprisingly, examples such as this have led to an even greater loss of trust in experts.

One of the most important and sustainable effects of the EBM initiative was, and still is, the advancement of methods to synthesize the available evidence systematically and transparently. The results of single studies are often imprecise, and the results of different studies on the same object may differ substantially. Systematic reviews and meta-analyses allow entire bodies of evidence to be assessed. The Cochrane Collaboration and independent researchers working outside the Cochrane Collaboration 
deserve much credit for developing and critically improving methods to synthesize evidence from different sources. Today, meta-analyses are performed by combining data not only at the level of study results, but also at the level of individual patients. Network meta-analyses are a recent advancement in the field; they assess the relative effectiveness of different treatments that have not been compared directly in randomized controlled trials [5]. Systematic reviews are meant to support "busy clinicians seeking bottom lines," so that they will not have to rely upon reviews by so-called experts or information sponsored by manufacturers of drugs/medical devices and can find "brief but valid summaries of best evidence" [2].

When we take a critical look at the promises made by proponents of EBM, we see that they have only partly been fulfilled. Clinicians and policy makers do not use systematic reviews as often as expected. Systematic reviews are often too long and detailed for busy clinicians to read; additionally, they do not provide the clinician with the relevant information. A survey performed by Glasziou et al. revealed that fewer than $15 \%$ of the systematic reviews published in Evidence based medicine provided sufficient information about the intervention to allow clinicians to implement it [6]. The conclusions of systematic reviews often state that the available evidence is inconclusive and that further studies must be performed. This is good news for researchers, but not very helpful for physicians caring for patients. The following is a typical example: the authors of a systematic review wanted to evaluate whether resection of the colon should be done primarily or as a staged procedure in patients with obstruction from left colorectal carcinoma. The main result reported by the authors reads "We identified 2,043 citations. One study for potential inclusion was identified, but was then excluded." The conclusion was " the limited number of identified studies ... do not allow a reliable assessment of the role of either therapeutic strategy ... it would appear advisable to conduct high quality large scale RCT to establish which treatment is more effective" [7].

Without a doubt, most researchers performing systematic reviews search carefully for the relevant literature in different databases, contact researchers to retrieve information not reported in the published papers, and try to obtain unpublished studies from drug and medical device companies. However, despite methodological advancements in merging primary study results into systematic reviews, major problems persist. Varying enrollment criteria in primary studies, differences in the definition and assessment of clinical outcomes, and the measurement of the outcome at varying time points hamper the pooling of different studies. The consequence is that even the results of systematic reviews on one and the same topic may be inconsistent.
This problem is exemplified in the article by Peterson and Hodler in this journal on the use of therapeutic injections for the spine and sacroiliac joints. A systematic review evaluating the benefit of injections in the facet joints of the lumbar spine concluded that there is moderate evidence that this therapy is effective in providing pain relief. Two other reviews came to the conclusion that there is no strong evidence for or against the use of injection therapy. Such inconsistencies are a matter of fact. For physicians and policy makers, such conflicting results and conclusions are not helpful; nor is the recommendation that further studies should be done.

Therefore, the solution is to establish a knowledge base of medicine from which physicians can draw the information that they need to care for patients [8]. A collection of evidence in the form of systematic reviews and the results of randomized trials or other clinical studies may not be sufficient. The crucial step is the transformation of evidence - the results of research - into knowledge. This is a task that should be undertaken by experts. Experts in a clinical specialty or subspecialty, in cooperation with experts in research methodology and statistics, would be competent to establish this knowledge base.

A prevailing characteristic trait of the EBM movement is the ambiguous and occasionally negative attitude toward expert opinions. Various scales for grading the levels of evidence have been developed and disseminated within the past decade. To my knowledge, in all of them, expert opinions lag behind single case reports in terms of trustworthiness. According to the recommendations of the Oxford Centre for Evidence-Based Medicine [9] regarding studies of prognosis, the highest level of evidence is a systematic review of randomized controlled trials, followed by single randomized trials, and cohort and other study designs. Expert opinions follow poor-quality cohort studies.

The categorization and grading of evidence levels is based on study design, and experts do not belong in this category. An expert should be a person of integrity with a high degree of knowledge about a certain subject. Expert opinions cannot be a substitute for the results of a randomized controlled trial or other carefully planned and executed studies, but expert knowledge is essential when an entire body of evidence is being assessed and evidence, the results of research, is being transformed to knowledge. The result of this process would be the knowledge base of medicine, which physicians could trust and rely upon. Knowledge in this context is defined as the shared beliefs of experts.

Carefully planned and executed primary studies and systematic reviews are a first and essential step in establishing the knowledge base, but the process should not stop there. I propose that a panel of experts in a specific field, for example, diagnostic and therapeutic injections for 
patients with joint and back pain, come together to appraise, discuss, and weigh the available evidence and then draw up a 'shared expert opinion.' The purpose of such a panel would not be to reach consensus on the least common denominator, and the desired result would not be a recommendation for what physicians should do. The result could be a publication, including a detailed description, as specific as possible, about the consequences in terms of probabilities. For example, the outcome of injections in the sacro-iliac joint of patients with chronic low back pain below L5 would be compared to the outcomes of other treatment modalities. This panel would provide knowledgeoriented guidance rather than action-oriented guidelines telling physicians and/or patients what they should do. Physicians would be able to provide patients with valuable information, and patients would be empowered to make informed decisions.

Specific considerations for further research could be an additional achievement of the panel. An obvious difficulty when one is evaluating the effect of treatment on patients with low back pain concerns the question of which patients to enroll in studies. In contrast with patients who have had a myocardial infarction, many patients with low back pain have structural or functional anomalies underlying their symptoms that are unknown. Researchers are trying to overcome this difficulty by identifying variables that will allow them to characterize these patients more accurately. One treatment modality is an injection of analgesics into the sacro-iliac joint or the surrounding structures in patients with chronic low back pain below L5. The rationale is that patients for whom pain decreases or disappears after the analgesics injection have a higher probability of benefiting from a therapeutic injection with steroids into the same joint. Comparisons of different studies reveal that no uniform technique was used and that different criteria were applied to judge whether pain arose from the sacro-iliac joint or elsewhere [10]. Therefore, those patients that were included in the studies assessing the effect of therapeutic injections with steroids might have differed with respect to the underlying causes of low back pain. Shared recom- mendations from experts regarding which test to utilize and how to measure the effect of diagnostic injections would be helpful for directing the planning and execution of further trials. In future systematic reviews, this will make it easier for study results to be compared and pooled.

Evidence is not synonymous with knowledge. Evidence is the result of research, and knowledge is, when based on reliable evidence, "shared expert beliefs." After the shunning of experts and authorities by the EBM movement, it is time to allow experts-impartial, objective persons of integrity with a high degree of knowledge in their fieldto return and establish the knowledge base of medicine.

\section{References}

1. Evidence-Based Medicine Working Group, Guyatt G, Cairns J, Churchill D, Cook D, Haynes B, et al. Evidence-based medicine. A new approach to teaching the practice of medicine. JAMA. 1992;268:2420-5.

2. Sackett DL, Richardson WS, Rosenberg W, Haynes RB. Evidence-based medicine. How to practice and teach EBM. New York: Churchill Livingstone; 1997.

3. Oxman AD, Guyatt GH. The science of reviewing research. Ann N Y Acad Sci. 1993;703:125-33.

4. Stelfox HT, Chua G, O'Rourke K, Detsky AS. Conflict of interest in the debate over calcium-channel antagonists. N Engl J Med. 1998;338:101-6.

5. Lumley T. Network meta-analysis for indirect treatment comparisons. Stat Med. 2002;21:2313-24.

6. Glasziou P, Meats E, Heneghan C, Shepperd S. What is missing from descriptions of treatment in trials and reviews? BMJ. 2008;336:1472-4.

7. De Salvo GL, Gava C, Lise M, Pucciarelli S. Curative surgery for obstruction from primary left colorectal carcinoma: Primary or staged resection? Cochrane Database Syst Rev. 2004;(2): CD002101.

8. Miettinen OS. Knowledge base of scientific gnosis: IV. Knowledge base of scientific gnosis vis-a-vis evidence base of this. J Eval Clin Pract. 2004;10:365-7.

9. Howick J. Oxford Centre for Evidence-Based medicine. Levels of evidence. 2009. http://www.cebm.net/index.aspx?o=1025. Accessed 8 September 2009

10. Rupert MP, Lee M, Manchikanti L, Datta S, Cohen SP. Evaluation of sacroiliac joint interventions: a systematic appraisal of the literature. Pain Physician. 2009;12:399-418. 